\title{
Chemometric Characterization of Chemlali Extra-Virgin Olive Oil Adulteration Mixed with Soybean Oil, Corn Oil and Sunflower Oil
}

\author{
Malika Drira $1^{a,}$ Hazem Jabeur ${ }^{a}$ and Mohamed Bouaziz $2^{a, b}$
}

${ }^{a}$ Laboratoire d'Electrochimie et Environnement, Ecole National d'Ingénieur de Sfax, Universitéde

Sfax, BP “1173”, 3038 Sfax, Tunisia

${ }^{b}$ Institut Supérieur de Biotechnologie de Sfax, Université de Sfax, BP “1175”, 3038 Sfax, Tunisia.

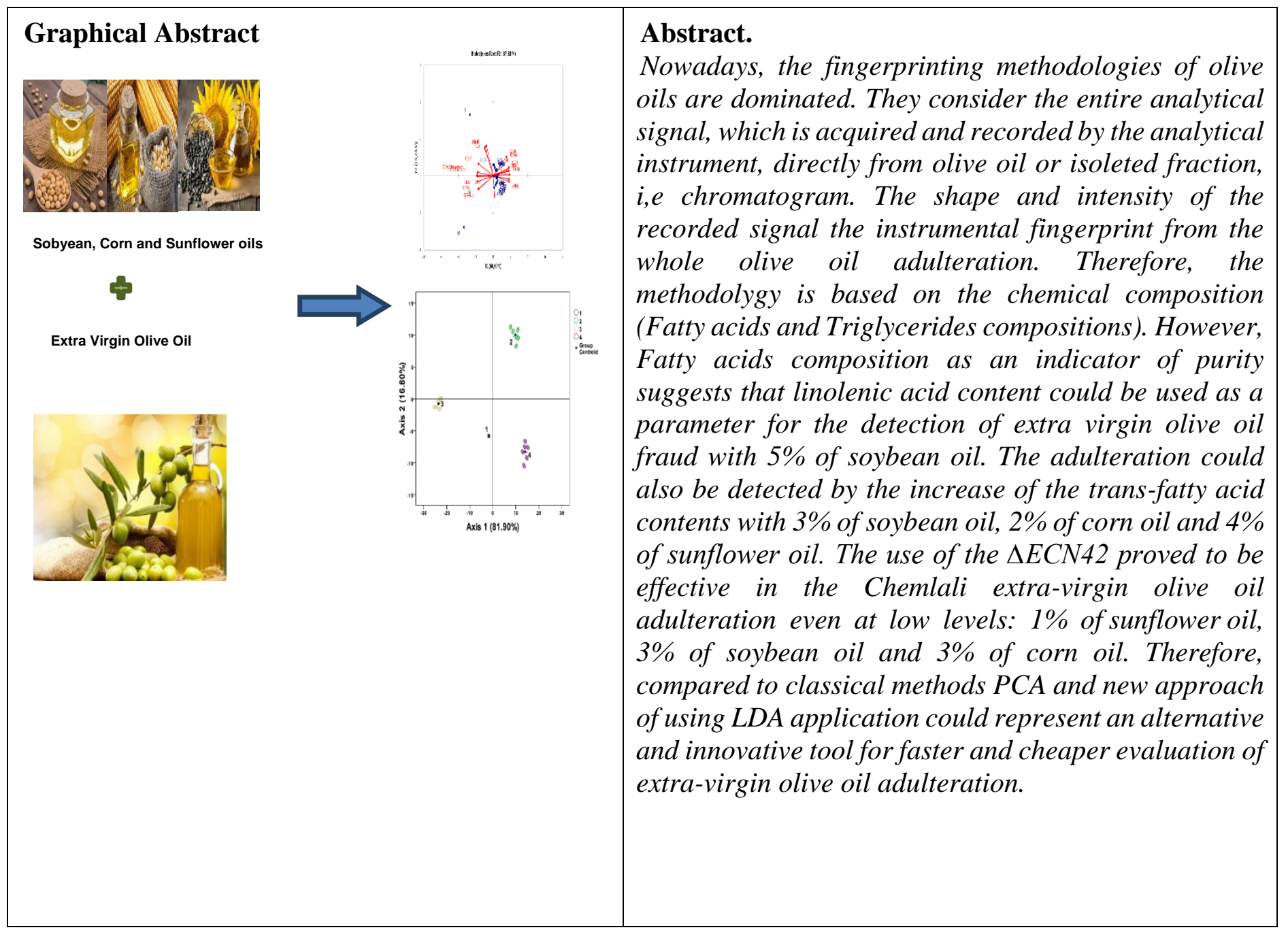

\section{Introduction}

Olive cultivation is widespread throughout the Mediterranean region and is important for the rural economy, local heritage and environment. In Tunisia, the olive oil sector plays an important role in the economy, providing both employment and export revenue. However, two important varieties dominate such as Chetoui and Chemlali. ${ }^{1,2}$ In fact, extra-virgin olive oil is usually more expensive than other vegetable oils for some reasons. It is also the oil that has not undergone any other treatment than washing, decantation, centrifugation and filtration. ${ }^{3}$ Therefore, different methods have been developed to control the falsification of the product authenticity and quality that is being perpetrated. The determinations of fatty acids and triacyglycerols(TAGs) through 
composition..$^{4-8}$

Fatty acid methyl esters (FAME), obtained by alkali/catalyzed transesterification of vegetable oils can be characterized by gas chromatography. ${ }^{9-12}$ The methyl esters of the fatty acids under investigation are usually the molecules used for the chemical analyses establishing the limits pertaining to the content of fatty acids in olive oil that can be used for the differentiation between genuine olive oil and other vegetable oils (soybean, sunflower and corn. $)^{13}$

TAGs are the main component of vegetable oils as they are generally found between the 97 to $99 \%$ range of the whole oil composition. The High Performance Liquid Chromatography (HPLC) quantitative analysis of TAGs are considered as an effective method for the detection of extra-virgin olive oil adulteration. ${ }^{14,15}$ The advantage of using TAGs profile includes the distribution of fatty acids between the different stereospecific positions on the glycerol molecule. TAGs are separated according to the equivalent carbon number (ECN) and the positions of double bond(s). Until recently, the most prominent methods to detect the adulteration of extra-virgin olive oil with other vegetable oils have been the trilinolein (LLL) content and the difference between the theoretical value of TAGs with an equivalent carbon number of 42 (ECN42theoretical). An appropriate software is used to compute the $\triangle E C N 42$ based on data of fatty acids composition and analytical triglyceride results (ECN42HPLC). ${ }^{16,17}$ Zeng et al. ${ }^{18}$ suggested the applicability of liquid chromatography electrospray tandem mass spectrometry (LC-ESI-MS) for the structural elucidation of TAGs in vegetable oils. However, the technique is able to resolve triglycerides both by atom carbon number and by unsaturation using phenyl-methyl-silicone stationary phases. ${ }^{19,20}$

Many publications report the application of the chemometrics such as the pricncipal component (PCA) and linear discriminant analysis (LDA) tools to find the best approach for the authenticity and traceability of extra-virgin olive oils. ${ }^{21-23}$

The utmost objective of the present research study has focused on discriminating and detection of the Chemlali extra-virgin olive oil adulteration with soybean, corn and sunflower oils. For this purpose, analyses of fatty acid and triacylglycerol profiles are performed using gas and liquid chromatography. This leads to the determination of the minimum detectable levels of vegetable oils (soybean, corn and sunflower).

\section{Materials and Methods}

\section{Vegetable Oils}

Four different extra-virgin olive oil samples were obtained from a Tunisian olive variety which is the Chemlali cultivar harvested from Sfax region (south Tunisia) during two crop seasons (2012/2013 and 2013/2014) (n=4; two different samples for each crop season).The ripening degree was the same for the four Chemlali olive samples (maturation indices were 4.5). The olive samples were collected at the beginning of December from orchards in the same neighborhood carried out with the same cultural practices. Pure soybean, corn and sunflower oils were purchased from the local market in Tunisia, used as adulterant, were checked for their authenticity by classical tests such as gas chromatography (fatty acid), with some being based on high-performance liquid chromatography (triglyceride composition). The different mixtures of extra-virgin olive oil with the aforementioned vegetable oils (at the levels of 1, 2, 3, 4, 5 and10\% w/w) were prepared.

\section{Determination of Fatty Acids Composition}

The methyl esters for the determination of the cis/ and trans-fatty acids was determined by Gas chromatograph equipped with a FID detector (HP 6890N, Agilent (Palo Alto, CA, USA)). According to the method reported by Jabeur et al., ${ }^{24}$

\section{Determination of Triglycerides Composition}

A $5 \%$ of the sample to be analyzed was prepared by weighing $0.25 \pm 0.001 \mathrm{~g}$ into a $5 \mathrm{~mL}$ graduated flask and dissolved in $5 \mathrm{~mL}$ with acetone. ${ }^{17}$ A HP1100 chromatograpic system from Agilent 
(wataoronn, ow, Germany) equippea with a aljerentat rejractometer aetector was empioyea.

Next, the separation was carried out with a spherisorb analytical column $(250 x 4.6 \mathrm{~mm}, 5 \mu \mathrm{m}$ particle size) from Supelco (Bellefonte, PA, USA). The optimized separation conditions were conducted by isocratic elution with a 60:40 acetone / acetonitrile mixture; column temperature, 30 ${ }^{\circ} \mathrm{C}$; flow rate, $1.5 \mathrm{~mL} / \mathrm{min}$ and injection volume, $20 \mu \mathrm{L}$ of the sample solution prepared as indicated above.

For the identification of TAGs, the retention times plotted in accordance with alternatively reference chromatograms obtained from soybean oil, mixture 30:70 soybean oil - olive oil and pure olive oil as described by COI. ${ }^{22}$ It was assumed that the sum of the areas of the peaks corresponding to the various TAGs was equal to $100 \%$, and the relative percentage of each TAGs was calculated. It is worthwhile to note that the theoretical value of ECN42 triacylglycerols was calculated by the computer programme.

\section{Statistical Analysis}

The results were expressed as mean \pm standard deviation (SD) of four measurements for the analytical determination. PCA and LDA was applied to discriminate Chemlali extra-virgin olive oil and the adulterated extra-virgin olive oils mixed with different percentages of soybean, corn and sunflower oils (1, 2, 3, 4, 5 and 10\%) according to all the parameters investigated. Both, PCA and LDA plots were performed using SPSS Statistics 17.0 for Windows (SPSS Inc., 2008).

\section{Results and Discussion}

\section{Identification of EVOO Adulteration with Other Low Cost Oils}

This research study is meant to detect adulteration of EVOO by lower cost seed oils. Consequently, various blends of EVOO and soybean, corn or sunflower oil were prepared and analyzed for fatty acid and triglyceride compositions. The adulteration percentages ranged from 1 to $10 \%$ in order to determine a threshold of detection.

\section{Use of the Fatty Acid and Triglyceride Compositions for the Detection of Fraud}

Fatty acid and TAG compositions of the adulterated extra-virgin olive oil mixed with 1-10\% (w/w) quantities of soybean, corn and sunflower oils were summarized in Figures 1, 2.

Taking into account the results presented in Figures 1, it could be concluded that the analysis offatty acids does not produce satisfactory results with regard to the levels of adulteration investigated in this research study. The most effective parameters for the detection of adulteration are mentioned below. The linolenic acid percentage could be used as a parameter for the detection of EVOO fraud with 5\% of soybean oil as well as by the increase of trans-fatty acid contents with $3 \%$ of soybean oil, $2 \%$ of corn oil and 4\% of sunflower oil (Table 2 and Figure 3). None of the other fatty acids is effective for the detection of the added vegetable oil, in an extra-virgin olive oil.

Although the composition of fatty acids in the examined seed oils is different from that of olive oils, the fatty acids experiment could not be satisfactorily used as discriminatory parameters between olive oil and the respective vegetable oil in most cases. According to Figure 2, it can be noted that the appearance of a specific triacylglycerol, normally not present in the olive oil, in oils adulterated with seed oils has a number of equivalent carbons equaling 40, known by LLLn. Thus, this triacylglycerol is an indicator of the presence of seed oil in extra-virgin olive oil. Indeed, 
LLLn increases Wun the increase in the aauteranon percenage of vegelable ous (Jrom 10

10\%). It ranges from 0.27 to $0.76 \%$ for the extra-virgin olive oil adulterated with soybean oil, from 0.11 to $0.69 \%$ for the extra-virgin olive oil adulterated with corn oil and from 0.05 to $0.11 \%$ for the extra-virgin olive oil adulterated with sunflower oil. The adulteration with soybean oil produces a large increase of the areas of peak LLLn because the soybean oil is the only one rich in linolenic acid.

The addition of small quantities of seed oil can be identified in the extra-virgin olive oil by the determination of the rate of LLL since the three seed oils are rich in linoleic acid (C18:2). Similarly, the presence of soybean, sunflower and corn oils were proven by an increase of the percentage of LLL and ECN42. The extra-virgin olive oils adulterated by the sunflower, corn and soybean oils (from 1 to 10\%). Having a content of LLL, they varied from 0.76 to $2.46 \%$ with sunflower oil, from 0.52 to $1.86 \%$ with corn oil and from 0.54 to $1.86 \%$ with soybean oil. Besides, having a practical ECN42, they varied from 1.26 to $3.39 \%$ with sunflower oil, from 1.33 to $2.87 \%$ with corn oil and from 1.42 to $3.59 \%$ with soybean oil. It is to be noted that olive oil is largely compared to the seed oils known by their highest linoleic acid percentages. The use of the $\triangle E C N 42$ was proved to be more effective in detecting even low levels of adulteration of extra-virgin olive oil with most of the examined vegetable oils. According to the data on the fraudulent mixtures presented in Figures1, the determination of the $\triangle E C N 42$ can be used as a parameter for the detection of fraud of extra-virgin olive oils with each one of the studied seed oils, $1 \%$ of sunflower $(0.21>0.20), 3 \%$ of soybean $(0.30>0.20)$ and $3 \%$ of corn $(0.21>0.20)$ (Table 2). This finding can be attributed to the fact that the $\triangle E C N 42$ is a number calculated by the combination of fatty acids and triglyceride composition. So, the difference in the composition of the triglycerides and the six fatty acids, taken into account for the calculation of the theoretical ECN42, between the initial samples and their mixtures can be expected. However, these differences do not have the expected effect on the values of the theoretical ECN42 and $\triangle E C N 42$. A consequence of this is the complete lack of correlation between linoleic acid or LLL and $\triangle E C N 42$ of vegetable oils and their admixtures with olive oil. These results are in agreement with those reported by Christopoulou et al., ${ }^{5}$ who claimed the parameter $\triangle E C N 42$ is very useful and effective in the detection of adulteration of olive oils with vegetable oils. 


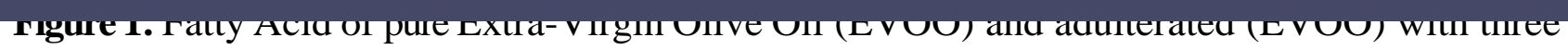
determinations: a: EVOO + (1-10\%) of soybean oil; b: EVOO + (1-10\%) of corn oil; c: EVOO + (1-10\%) of sunflower oil.

$\mathbf{a}$
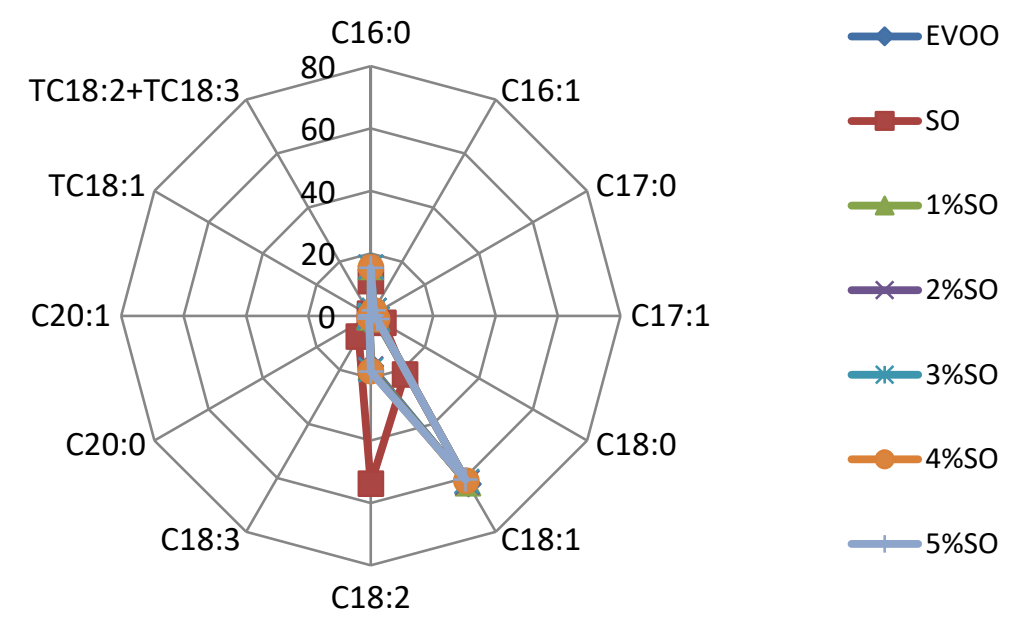

b
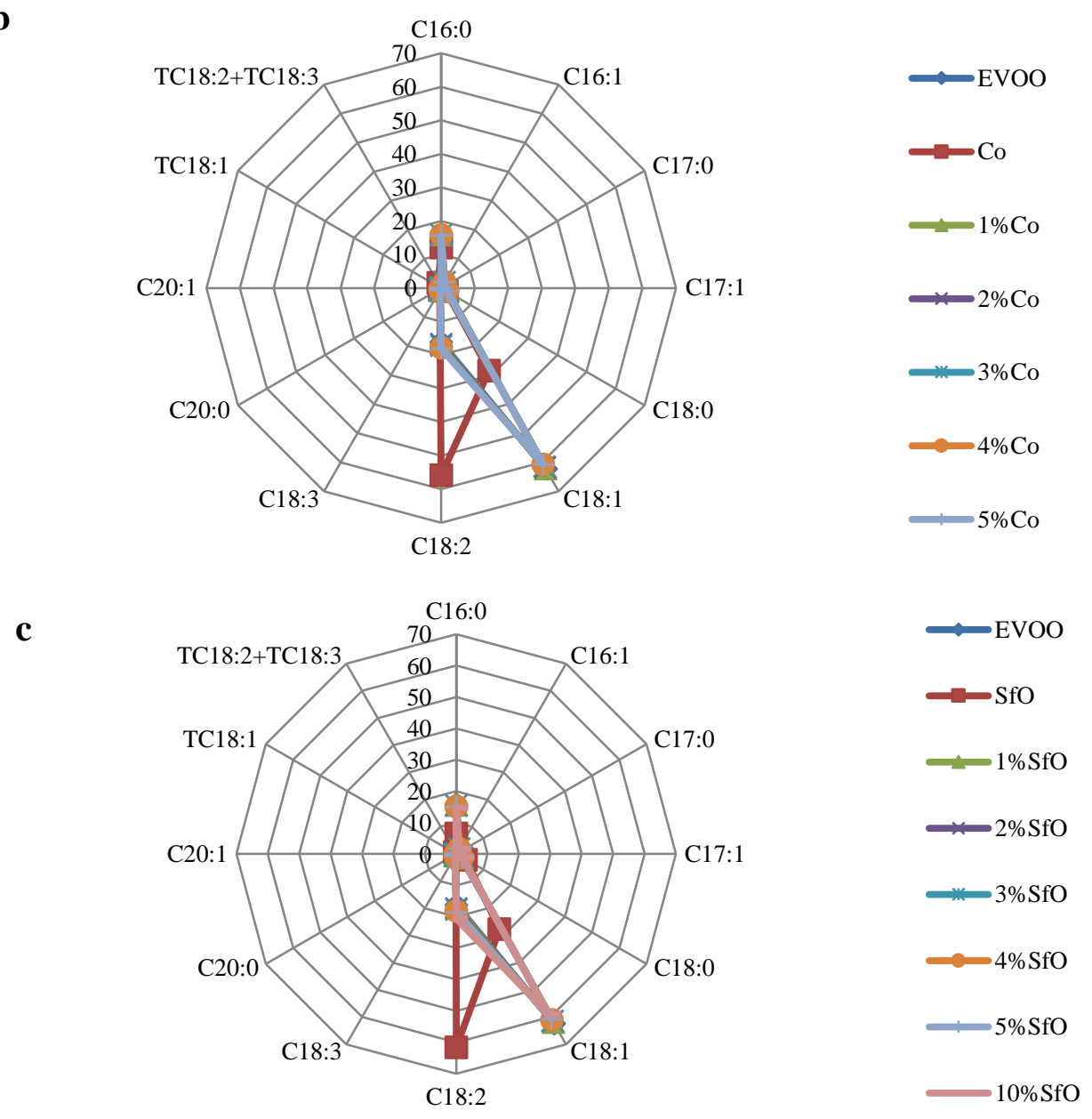

Figure 2. Triacylglycerol compositions of pure Extra-Virgin Olive Oil (EVOO) and adulterated (EVOO) with three determinations: a: EVOO + (1-10\%) of soybean oil; b: EVOO + (1-10\%) of corn oil; c: EVOO + (1-10\%) of sunflower oil. 
a
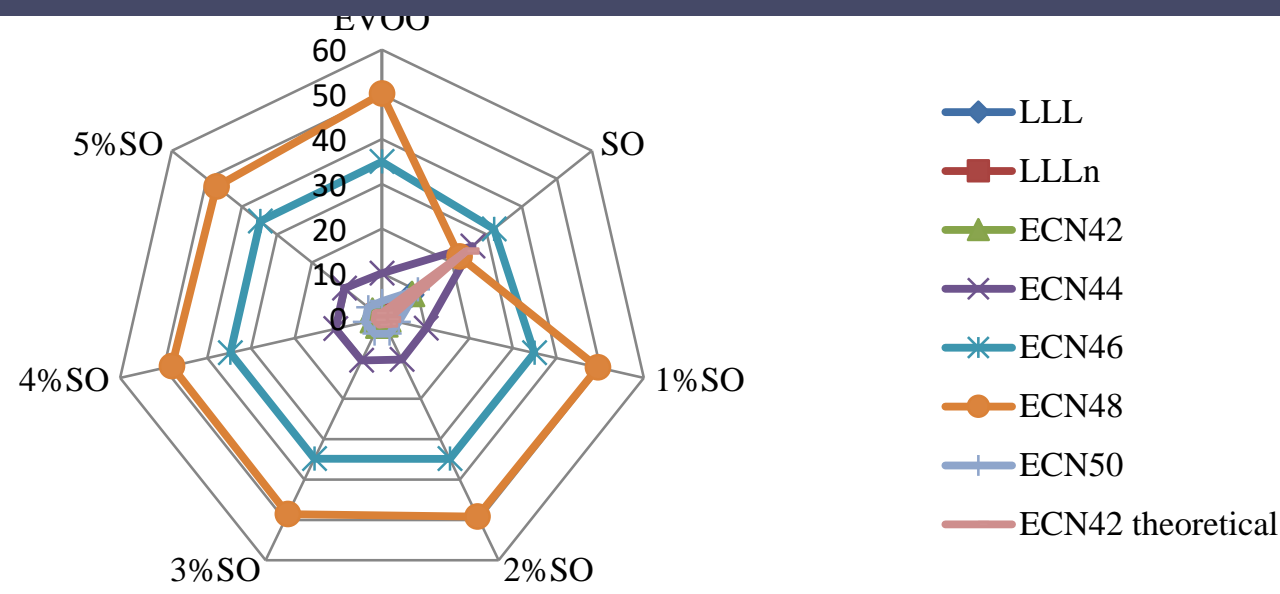

b
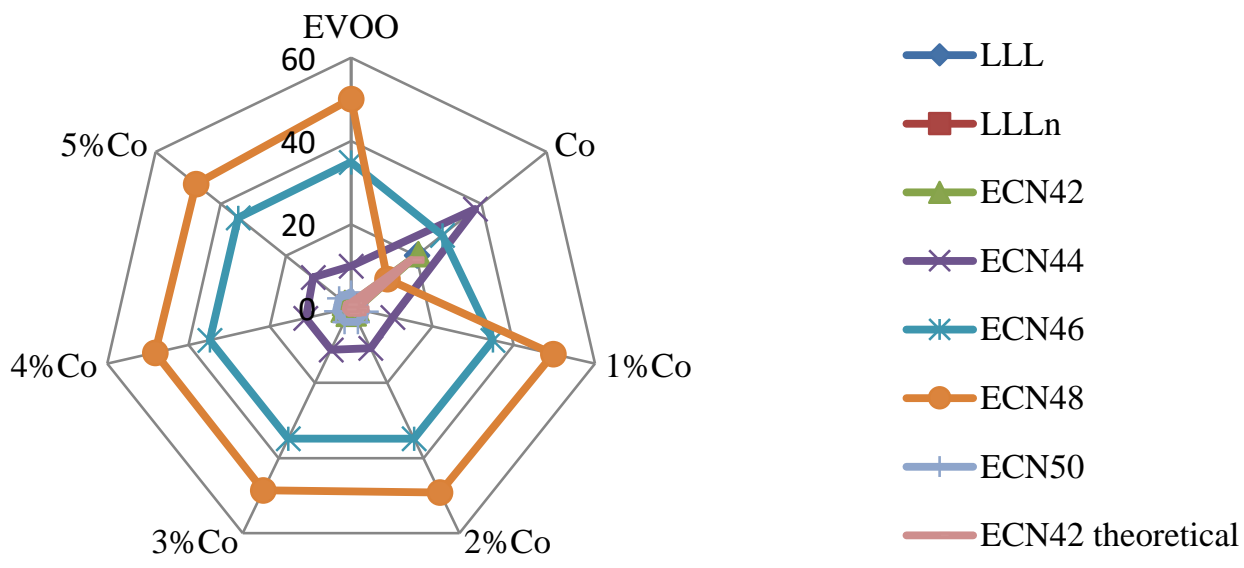

c

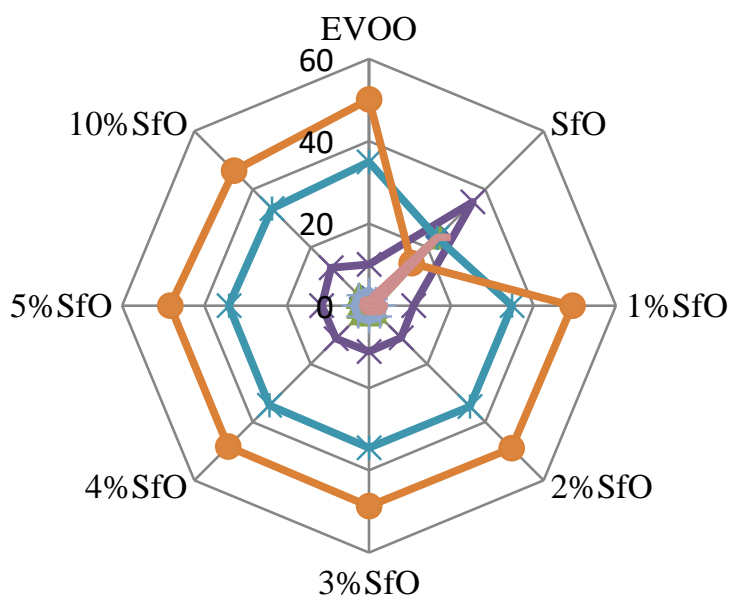

$\longrightarrow$ LLL

-LLLn

$\leftarrow$ ECN42

$\leftarrow$ ECN44

$\rightarrow$ ECN46

ECN48

+ ECN50

ECN42 theoretical

Figure 3. Trans-fatty acids of mixtures of EVOO with (a) soybean, (b) corn and (c) sunflower oils. Each value represents the mean of four determinations of two successive crop seasons ( $n=4$; Standard deviation < 0.001\%). EVOO: extra-virgin olive oil 

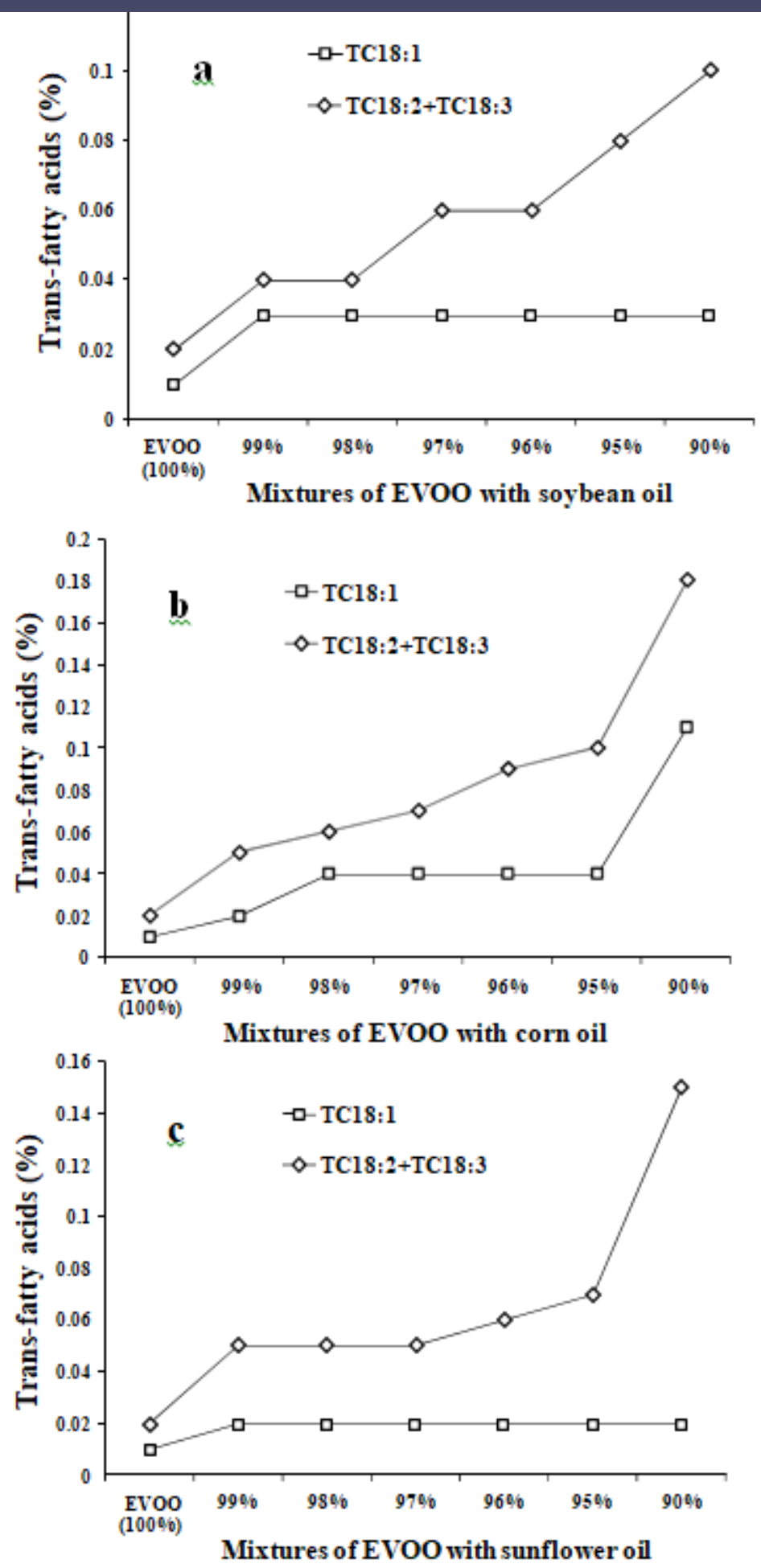

Table 2. Used Parameters for the Detection of Extra-Virgin Olive Oil Adulteration with Vegetable Oils 


\begin{tabular}{|c|c|c|c|}
\hline Type of seed & adulteration & $\begin{array}{l}\text { COI threshol } \\
\text { value }\end{array}$ & $\begin{array}{l}\text { the COI threshold } \\
\text { value }\end{array}$ \\
\hline \multirow[t]{7}{*}{ Soybean } & $\mathrm{C} 18: 3$ & $1 \%$ & EVOO+5\% SO (1.08\%) \\
\hline & ECN40 & 0 & EVOO+1\% SO (0.27) \\
\hline & $\triangle \mathrm{ECN} 42$ & 0.20 & EVOO+3\% SO (0.30) \\
\hline & Campesterol & $4 \%$ & EVOO+10\% SO $(6.17 \%)$ \\
\hline & $\Delta 7$-Stigmastenol & $0.50 \%$ & EVOO+10\% SO (0.59\%) \\
\hline & Apparent $\beta$-sitosterol & $93.00 \%$ & EVOO+10\% SO (89.21\%) \\
\hline & $\sum(\mathrm{TC} 18: 2+\mathrm{TC} 18: 3)$ & $0.05 \%$ & EVOO+3\% SO (0.06\%) \\
\hline \multirow[t]{5}{*}{ Corn } & ECN40 & 0 & EVOO+1\% CO (0.11) \\
\hline & $\triangle \mathrm{ECN} 42$ & 0.20 & $\mathrm{EVOO}+3 \% \mathrm{CO}(0.21)$ \\
\hline & Campesterol & $4 \%$ & EVOO+4\% CO (4.06\%) \\
\hline & Apparent $\beta$-sitosterol & $93.00 \%$ & $\begin{array}{l}\text { EVOO+10\% CO } \\
(91.86 \%)\end{array}$ \\
\hline & $\sum(\mathrm{TC} 18: 2+\mathrm{TC} 18: 3)$ & $0.05 \%$ & EVOO+2\% CO (0.06\%) \\
\hline \multirow[t]{5}{*}{ Sunflower } & ECN40 & 0 & EVOO+1\% SfO (0.05) \\
\hline & $\triangle \mathrm{ECN} 42$ & 0.20 & EVOO+1\% SfO (0.21) \\
\hline & $\Delta 7$-Stigmastenol & $0.50 \%$ & EVOO+1\% SfO $(0.54 \%)$ \\
\hline & Apparent $\beta$-sitosterol & $93.00 \%$ & $\begin{array}{l}\text { EVOO+5\% SFO } \\
(92.89 \%)\end{array}$ \\
\hline & $\sum(\mathrm{TC} 18: 2+\mathrm{TC} 18: 3)$ & $0.05 \%$ & EVOO+4\% SfO $(0.06 \%)$ \\
\hline
\end{tabular}

\section{Chemometric Analysis}

LDA is probably the most frequently used supervised pattern recognition method. In principle, LDA determines linear discriminantfunctions, which maximise the ratio of betweenclass variance and minimise the ratio of within-class variance. It should be noted that, whereas the principal component analysis (PCA) selects a direction that retains maximal structure among the data in a lower dimension (Figure 4), LDA selects a direction that achieves maximum separation among the given classes. ${ }^{25}$

Since the data structure analysis gave a good sample characterization, a classification model was built. LDA analysis was applied in order to find a predictive classification model, able to differentiate the pure extra-virgin olive oil and the adulterated olive oils.

The plot of the discriminant functions (Figure 5) obtained by LDA showed a clear discrimination between the Chemlali extra-virgin olive oil and the adulterated extra-virgin olive oils mixed with different percentages of soybean, corn and sunflower oils (1, 2, 3, 4, 5 and 10\%). Discriminant function 1, which was highly related to ECN50, LLLn, C17:1 and C18:0, represent the function of minor compounds, already considered as a fingerprint for specific oil. Discriminant function 2 is highly related to C17:0, ECN42theoretical, LLLn and C18:3. In particular, by discriminant function 1 it was possible to discriminate Chemlali extra-virgin olive oil and the adulterated extra-virgin olive oils whatever the percentage of seed oil added (Figure5). All samples were correctly discriminated using the two functions. Application of LDA, after feature selection, was sufficient to differentiate Chemlali extra virgin olive oil and all adulterated extra-virgin olive oils. The success was $100 \%$ in classification and close to $100 \%$ in prediction.

It is difficult to discriminate the adulterated EVOO with $1 \%$ of sunflower oil and the pure EVOO whereas those adulterated with other vegetables oils are clearly separated at the same level (Figure 6a). The separation improves when the percentage of adulteration increases (Figure 6b, $c$ and d). 
Comparea 10 ctasstcat metnoas, ints new approacn oJ ustng LDA applicalion coula represent an

alternative and innovative tool for faster and cheaper evaluation of extra-virgin olive oil adulteration.

Figure 4. PCA b i plot of pure EVOO and adulterated EVOO based on all the analyses performed with four determinations. 1: EVOO: extra-virgin olive oil; 2: EVOO + (1-10\%) of soybean oil; 3 : EVOO $+(1-10 \%)$ of corn oil; 4 : EVOO + (1-10\%) of sunflower oil.

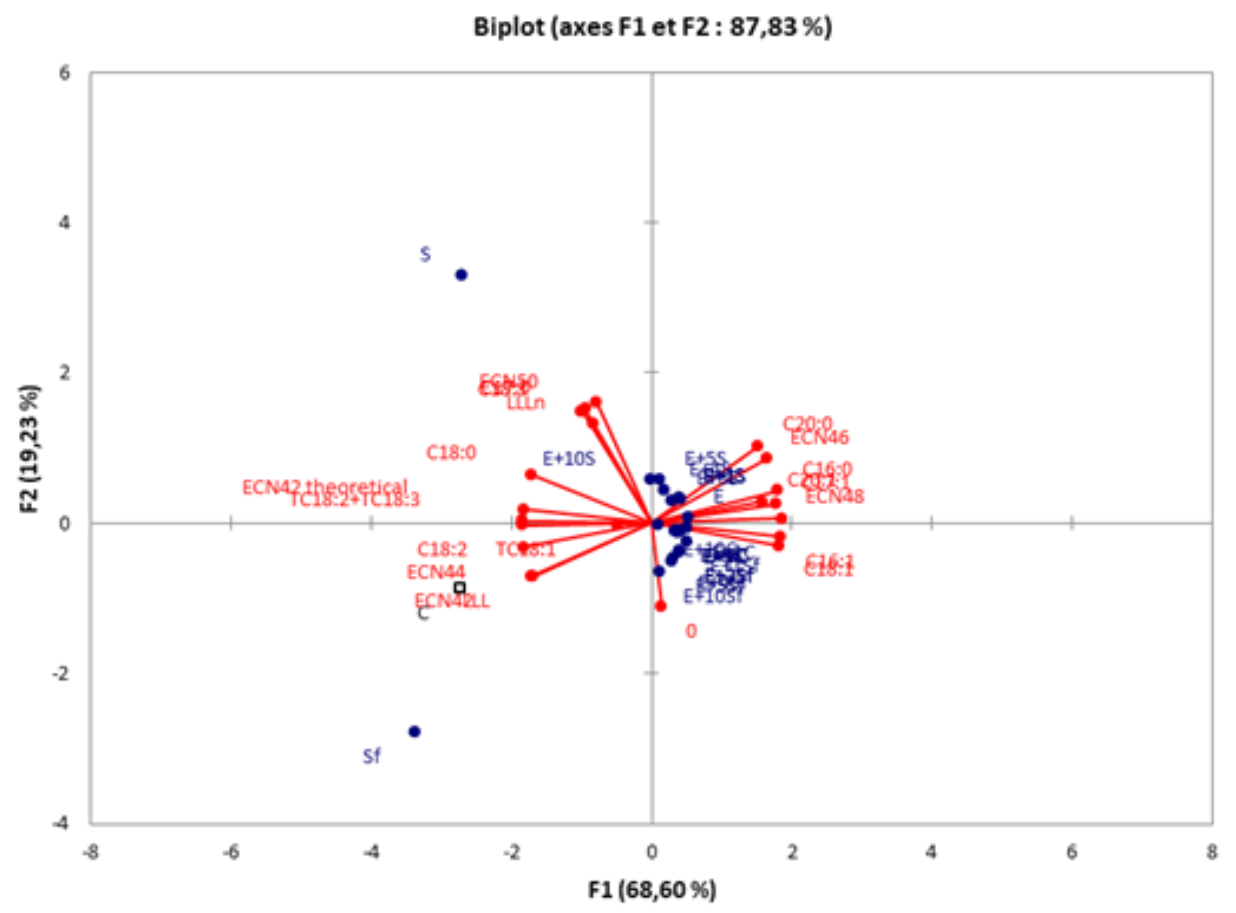

Figure 5. LDA score plot of pure EVOO and adulterated EVOO based on all the analyses performed with four determinations. 1: EVOO: extra-virgin olive oil; 2: EVOO $+(1-10 \%)$ of soybean oil; 3: EVOO + (1-10\%) of corn oil; 4: EVOO + (1-10\%) of sunflower oil. 


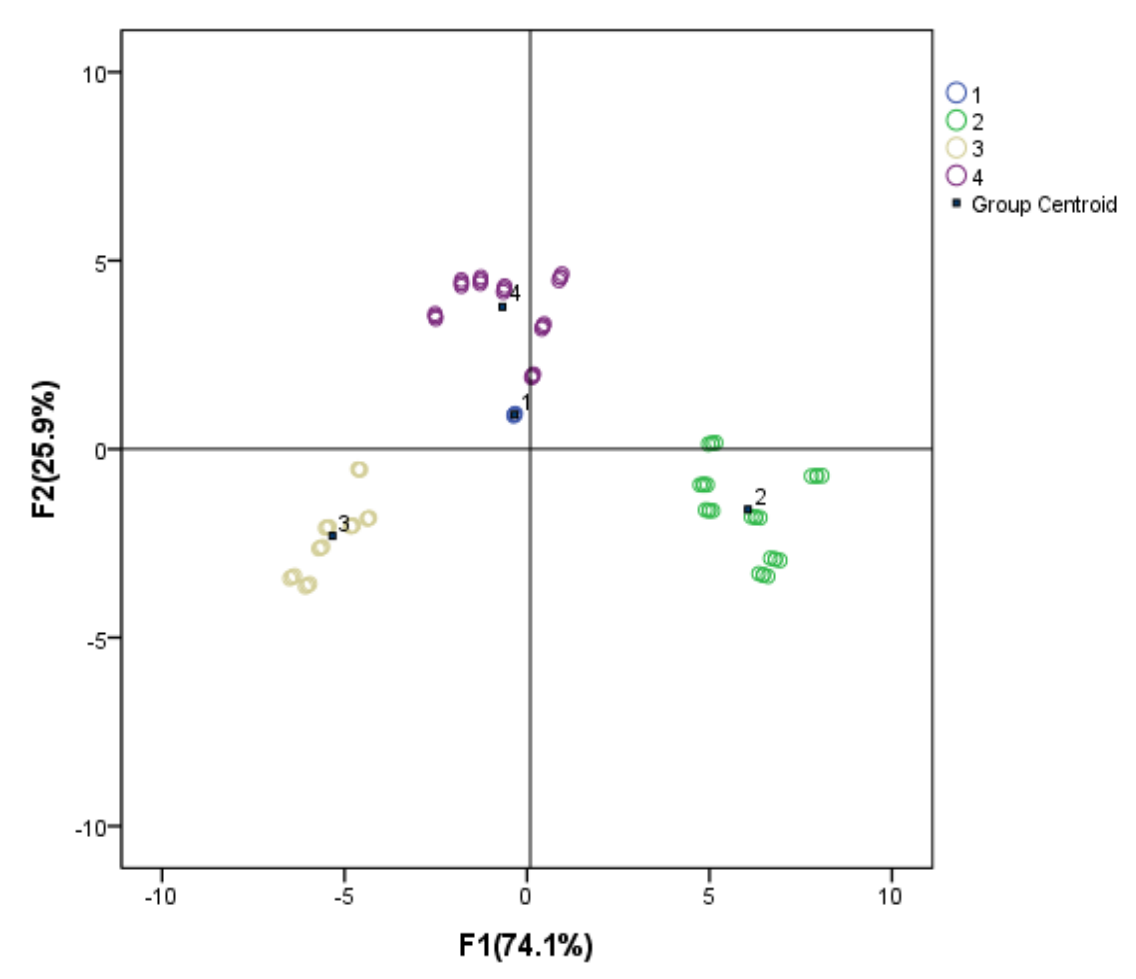

Figure 6. LDA score plot of pure EVOO, pure seed oils and adulterated EVOO at the same level based on all the analyses performed with four determinations. (a): EVOO $+1 \%$ of seed oil; (b): EVOO $+2 \%$ of seed oil; (c): EVOO $+5 \%$ of seed oil; (d): EVOO $+10 \%$ of seed oil. 1: EVOO: extra-virgin olive oil; 2: soybean oil; 3: corn oil; 4: sunflower oil; 5: EVOO + \% of soybean oil; 6: EVOO $+\%$ of corn oil; $7:$ EVOO $+\%$ of sunflower oil.

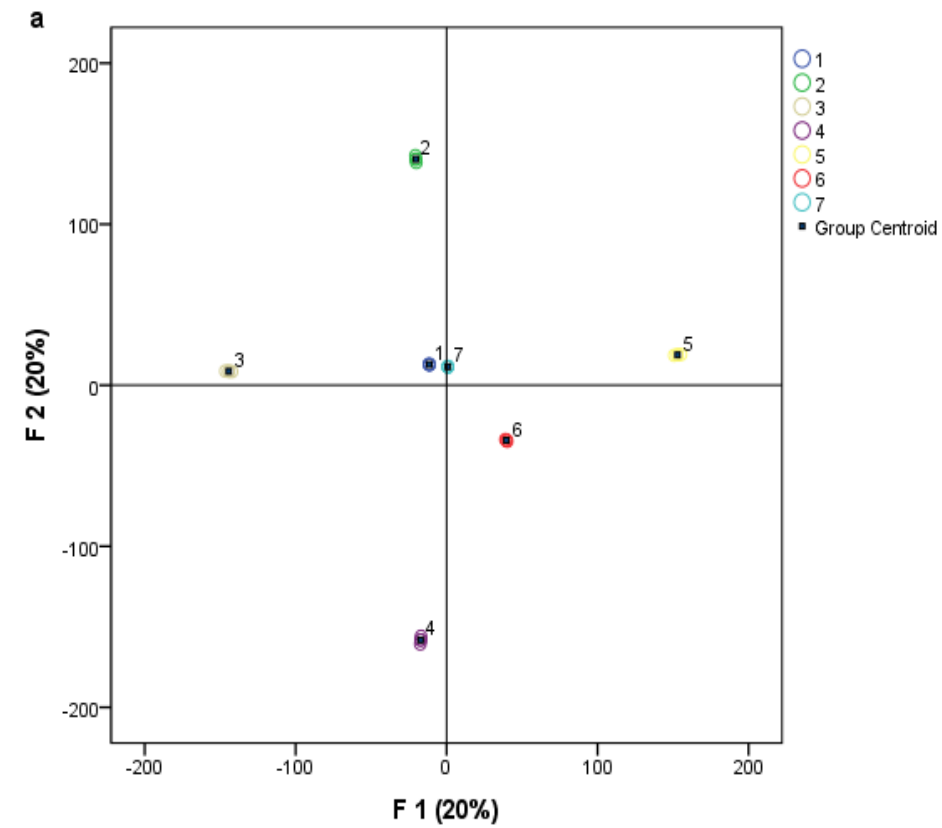



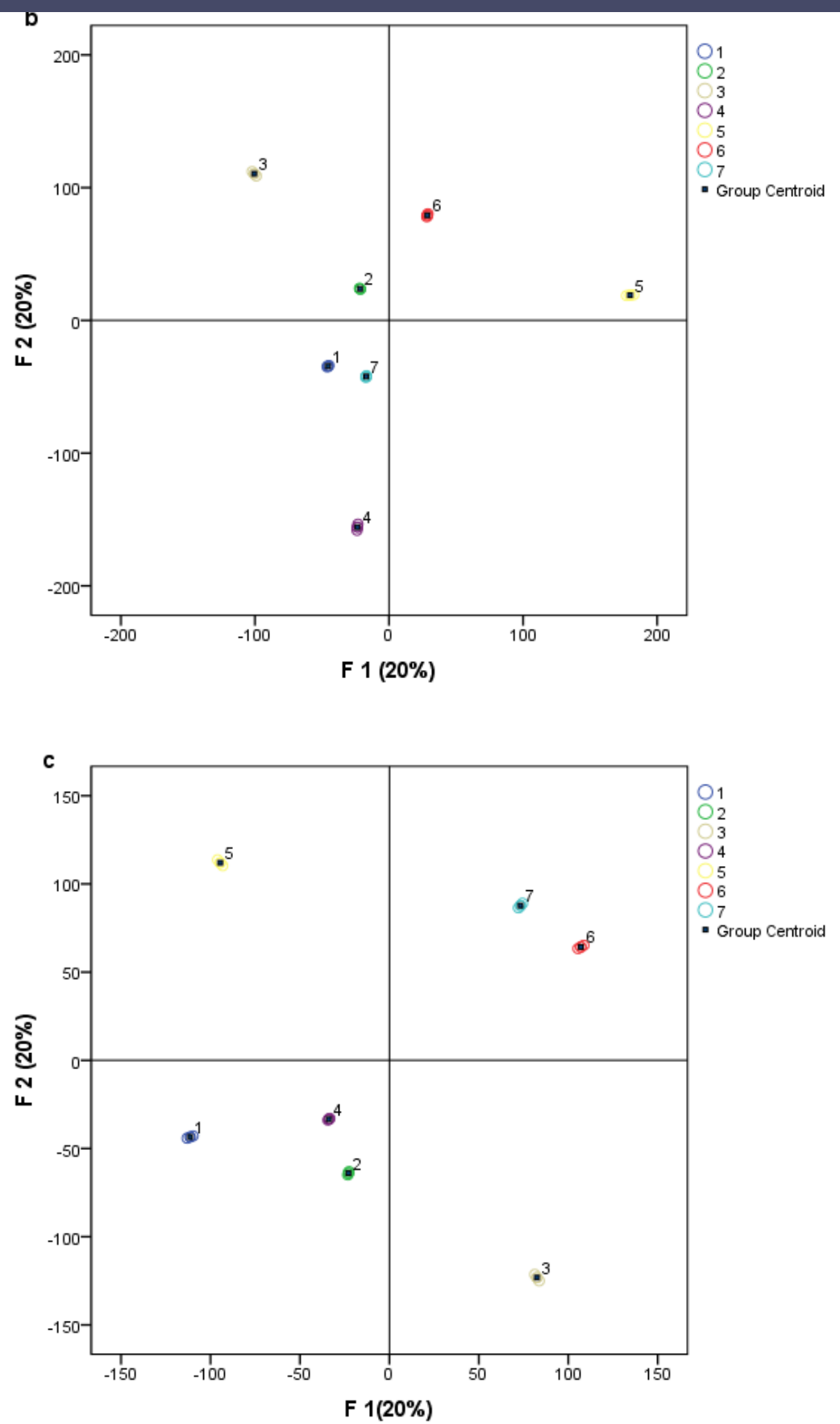


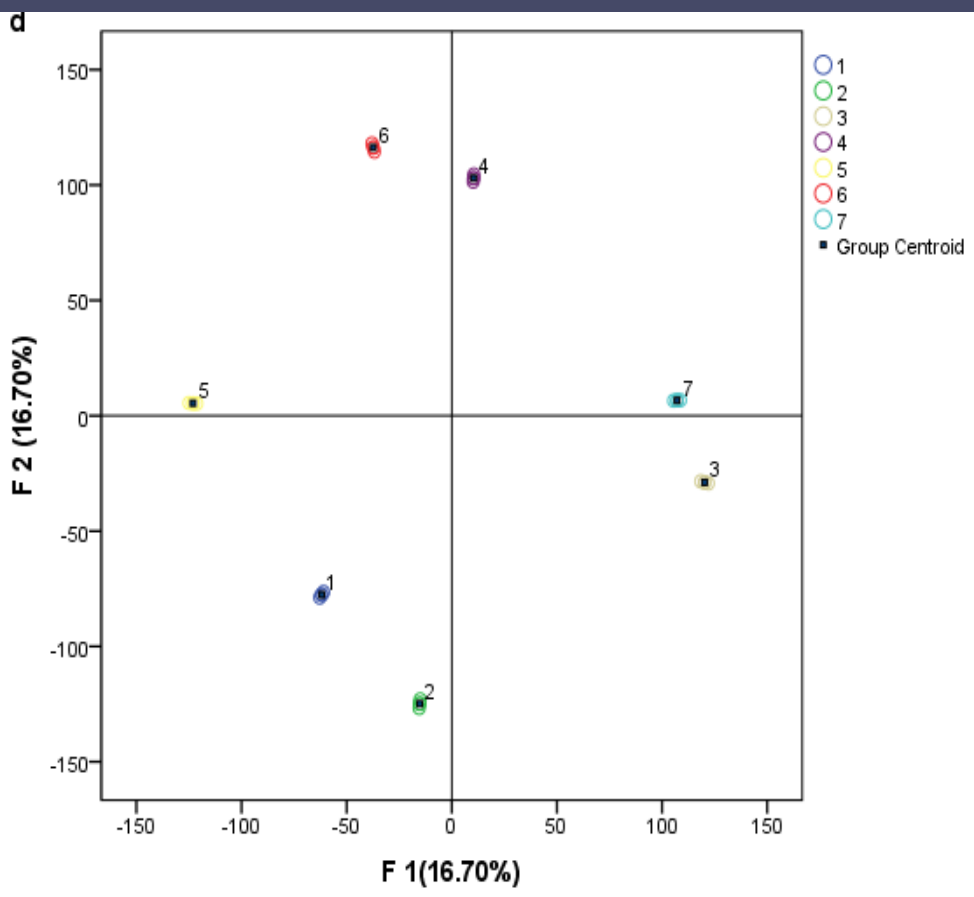

\section{Conclusions}

The present research, aimed at discriminate the Chemlali extra-virgin olive oils adulteration by some cheaper vegetable oils such as soybean, corn and sunflower oils, was detected using a gas chromatography in combination with liquid chromatography. According to these techniques, certain compounds found in the studied oils (triacylglycerols and fatty acids) are identified, analyzed and used for the detection of the adulteration of extra-virgin olive oils. It should also be focused on two multivariate analysis methods: principal component analysis (PCA) and linear discriminant analysis (LDA). This study has shown that LDA on chemical composition data can be used to separate the adulteration extra-virgin olive oil with the most common vegetable oils into groups.

\section{References}

(1) Haddada, F. M.; Manaï, H.; Oueslati, I.; Daoud, D.; Sánchez, J.; Osorio, E.; Zarrouk, M. Fatty Acid, Triacylglycerol, and Phytosterol Composition in Six Tunisian Olive Varieties. J. Agric. Food Chem. 2007, 55 (26), 10941-10946. https://doi.org/10.1021/jf071727a.

(2) Tena, N.; Lazzez, A.; Aparicio-Ruiz, R.; García-González, D. L. Volatile Compounds Characterizing Tunisian Chemlali and Chétoui Virgin Olive Oils. J. Agric. Food Chem. 2007, 55 (19), 7852-7858. https://doi.org/10.1021/jf071030p.

(3) International Olive Council. Trade Standard Applying to Olive Oils and Olive-Pomace Oils. 2013, COI/T.15/NC No 3/Rev. 7.

(4) Aparicio, R.; Aparicio-Ruíz, R. Authentication of Vegetable Oils by Chromatographic Techniques. J. Chromatogr. A 2000, 881 (1-2), 93-104.

(5) Christopoulou, E.; Lazaraki, M.; Komaitis, M.; Kaselimis, K. Effectiveness of Determinations of Fatty Acids and Triglycerides for the Detection of Adulteration of Olive Oils with Vegetable Oils. Food Chem. 2004, 84 (3), 463-474. https://doi.org/10.1016/S0308-8146(03)00273-5.

(6) Gurdeniz, G.; Ozen, B. Detection of Adulteration of Extra-Virgin Olive Oil by Chemometric Analysis of Mid-Infrared Spectral Data. Food Chem. 2009, 116 (2), 519-525. https://doi.org/10.1016/j.foodchem.2009.02.068.

(7) Cordella, C.; Moussa, I.; Martel, A.-C.; Sbirrazzuoli, N.; Lizzani-Cuvelier, L. Recent Developments in Food Characterization and Adulteration Detection: Technique-Oriented Perspectives. J. Agric. Food Chem. 2002, 50 (7), 1751-1764.

(8) Škevin, D.; Kraljić, K.; Miletić, L.; Obranović, M.; Neđeral, S.; Petričević, S. Adulteration of Oblica Virgin Olive Oil with Edible Sunflower and Refined Olive Pomace Oil. Croat. J. Food Technol. Biotechnol. Nutr. 2011, 6 (3-4), 117-122. 
Antioxidants. J. Agric. Food Chem. 2010, 58 (10), 5991-6006. https://doi.org/10.1021/jf1007677.

(10) Aparicio, R.; Aparicio-Ruíz, R. Authentication of Vegetable Oils by Chromatographic Techniques. J. Chromatogr. A 2000, 881 (1), 93-104. https://doi.org/10.1016/S00219673(00)00355-1.

(11) Garcés, R.; Mancha, M. One-Step Lipid Extraction and Fatty Acid Methyl Esters Preparation from Fresh Plant Tissues. Anal. Biochem. 1993, 211 (1), 139-143. https://doi.org/10.1006/abio.1993.1244.

(12) de la Mata-Espinosa, P.; Bosque-Sendra, J. M.; Bro, R.; Cuadros-Rodríguez, L. Olive Oil Quantification of Edible Vegetable Oil Blends Using Triacylglycerols Chromatographic Fingerprints and Chemometric Tools. Talanta 2011, 85 (1), 177-182. https://doi.org/10.1016/j.talanta.2011.03.049.

(13) Jabeur, H.; Zribi, A.; Makni, J.; Rebai, A.; Abdelhedi, R.; Bouaziz, M. Detection of Chemlali Extra-Virgin Olive Oil Adulteration Mixed with Soybean Oil, Corn Oil, and Sunflower Oil by Using GC and HPLC. J. Agric. Food Chem. 2014, 62 (21), 4893-4904. https://doi.org/10.1021/jf500571n.

(14) Tsimidou, M.; Macrae, R.; Wilson, I. Authentication of Virgin Olive Oils Using Principal Component Analysis of Triglyceride and Fatty Acid Profiles: Part 2-Detection of Adulteration with Other Vegetable Oils. Food Chem. 1987, 25 (4), 251-258. https://doi.org/10.1016/03088146(87)90011-2.

(15) Tay, A.; Singh, R. K.; Krishnan, S. S.; Gore, J. P. Authentication of Olive Oil Adulterated with Vegetable Oils Using Fourier Transform Infrared Spectroscopy. LWT - Food Sci. Technol. 2002, 35 (1), 99-103. https://doi.org/10.1006/fstl.2001.0864.

(16) García-González, D. L.; Viera, M.; Tena, N.; Aparicio, R. Evaluation of the Methods Based on Triglycerides and Sterols for the Detection of Hazelnut Oil in Olive Oil. Grasas Aceites 2007, 58 (4), 344-350. https://doi.org/10.3989/gya.2007.v58.i4.445.

(17) International Olive Council, COI/T, 20/Doc. No. 20 Rev. 3. Determination of the Difference between Actual and Theoretical Content of Triacylglycerols with ECN42. 2010.

(18) Zeng, Y.-X.; Araujo, P.; Du, Z.-Y.; Nguyen, T.-T.; Frøyland, L.; Grung, B. Elucidation of Triacylglycerols in Cod Liver Oil by Liquid Chromatography Electrospray Tandem Ion-Trap $\begin{array}{llllll}\text { Mass } & \text { Spectrometry. } & \text { Talanta } & \text { 2010, } & 82 & \text { (4), }\end{array}$ https://doi.org/10.1016/j.talanta.2010.06.055.

(19) Antoniosi Filho, N. R.; Carrilho, E.; Lanças, F. M. Fast Quantitative Analysis of Soybean Oil in Olive Oil by High-Temperature Capillary Gas Chromatography. J. Am. Oil Chem. Soc. 1993, 70 (10), 1051-1053. https://doi.org/10.1007/BF02543037.

(20) Geeraert, E.; Sandra, P. Capillary GC of Triglycerides in Fats and Oils Using a High Temperature Phenylmethylsilicone Stationary Phase. Part II. The Analysis of Chocolate Fats. $J$. Am. Oil Chem. Soc. 1987, 64 (1), 100-105. https://doi.org/10.1007/BF02546261.

(21) Longobardi, F.; Ventrella, A.; Napoli, C.; Humpfer, E.; Schütz, B.; Schäfer, H.; Kontominas, M. G.; Sacco, A. Classification of Olive Oils According to Geographical Origin by Using $1 \mathrm{H}$ NMR Fingerprinting Combined with Multivariate Analysis. Food Chem. 2012, 130 (1), 177183. https://doi.org/10.1016/j.foodchem.2011.06.045.

(22) Jabeur, H.; Drira, M.; Rebai, A.; Bouaziz, M. Putative Markers of Adulteration of Higher-Grade Olive Oil with Less Expensive Pomace Olive Oil by GC Combined with Chemometrics. $J$. Agric. Food Chem. 2017, 65. https://doi.org/10.1021/acs.jafc.7b00687.

(23) Bucci, R.; Magrí, A. D.; Magrí, A. L.; Marini, D.; Marini, F. Chemical Authentication of Extra Virgin Olive Oil Varieties by Supervised Chemometric Procedures. J. Agric. Food Chem. 2002, 50 (3), 413-418.

(24) Jabeur, H.; Zribi, A.; Makni, J.; Rebai, A.; Abdelhedi, R.; Bouaziz, M. Detection of Chemlali Extra-Virgin Olive Oil Adulteration Mixed with Soybean Oil, Corn Oil, and Sunflower Oil by Using GC and HPLC. J. Agric. Food Chem. 2014, 62 (21), 4893-4904. https://doi.org/10.1021/jf500571n.

(25) Berrueta, L. A.; Alonso-Salces, R. M.; Héberger, K. Supervised Pattern Recognition in Food $\begin{array}{llllll}\text { Analysis. } \quad J . & \text { Chromatogr. } & \text { A 2007, } & 1158 \quad \text { (1), 196-214. }\end{array}$ https://doi.org/10.1016/j.chroma.2007.05.024. 
